\title{
General expression of poly(methyl methacrylate) optical fiber Bragg grating sensing response
}

\author{
W. Zhang, Member, IEEE, and D. J. Webb, Member, IEEE
}

\begin{abstract}
Poly(methyl methacrylate) (PMMA) optical fiber inherently responds to the changes of temperature and relative humidity. PMMA optical fiber Bragg grating (POFBG) has been reported with diverse temperature and relative humidity sensitivities. These results cannot present the full performance of POFBG sensor as they are measured at limited environmental conditions. In this work we systematically investigated the POFBG temperature and relative humidity sensing responses. We found that POFBG temperature/relative humidity sensitivity is the function of both temperature and relative humidity. In the experiments POFBG sensing responses vary linearly with temperature/relative humidity at fixed relative humidity/temperature. Based on this linearity a general expression of POFBG sensing response to temperature and relative humidity has been obtained and verified for the first time, to describe the POFBG performance in desired operation range. The anisotropy in POF induced by drawing history could induce uncertainty in POFBG response but pre-straining POFBG overcomes this problem.
\end{abstract}

Index Terms - Fiber Bragg gratings, polymer optical fiber, temperature measurement, relative humidity measurement.

\section{INTRODUCTION}

$\mathrm{T}$ HE physical and chemical properties of polymeric materials are rather different to silica, potentially making it attractive for researchers to exploit in device and sensing applications. Polymer optical fiber gratings provide additional advantages for sensing applications including a much higher failure strain and a Young's modulus, biologically compatible with human tissues, negative refractive index (RI) change against temperature rise (the opposite of that for silica), affinity for water that leads to a swelling of the fiber and an increase of refractive index, etc. Bragg gratings have been inscribed into PMMA optical fiber (POF) in both step-index and microstructured geometries [1]. One of the interesting features of PMMA optical fiber gratings (POFBGs) is the negative refractive index change over temperature increase. It offers a well-conditioned performance for overcoming the crosssensitivity issues existing in silica fiber gratings [2]. Another

This work was supported in part by the European Commission Seventh Framework Programme under grant agreement no. 314032.

W. Zhang is with School of Aerospace, Transport and Manufacturing, Cranfield University, Cranfield, MK43 0AL, UK. (e-mail: zhang.wei@cranfield.ac.uk). feature of water affinity of PMMA has led to the sensing applications on relative humidity [3], saline concentration [4], water in aviation fuel [5], moisture in transformer oil [6].

POFBG can inherently be used as environmental sensor as it always responds to the changes of environmental temperature and relative humidity. There have been many reports on POFBG sensor's sensitivities of temperature and relative humidity. Despite considering different types of polymer optical fibers used, both temperature and relative humidity sensitivity of polymer optical fiber gratings reported are inconsistent and puzzling. From results reported in the literatures, the POFBGs made of different PMMA-based optical fibers exhibit different temperature sensitivities: for instance, $-55 \mathrm{pm} /{ }^{\circ} \mathrm{C}(50 \% \mathrm{RH})$ in $[2],-149 \mathrm{pm} /{ }^{\circ} \mathrm{C}$ (ambient condition) in [7], $-138 \mathrm{pm} /{ }^{\circ} \mathrm{C}$ (ambient condition) in [8], up to $-360 \mathrm{pm} /{ }^{\circ} \mathrm{C}$ (ambient condition) in [9]. In the $1550 \mathrm{~nm}$ region PMMA fiber gratings show the relative humidity sensitivity from $35 \mathrm{pm} / \% \mathrm{RH}$ (at $25^{\circ} \mathrm{C}$ ) [2] to nearly $80 \mathrm{pm} / \% \mathrm{RH}$ (at 22.2 $\left.{ }^{\circ} \mathrm{C}\right)[3,8]$. These reported figures are quite diverse. Most of them were measured by looking into POFBG wavelength response over, e.g., varying temperature at ambient condition without setting or controlling relative humidity (or varying humidity without controlling temperature). However, ambient condition varies during experiment so that the obtained POFBG temperature sensitivity is the result over an unknow range of relative humidity perturbation. In some case POFBG temperature sensitivity is investigated while POFBG is mounted on Peltier device to change its temperature. In this occasion when temperature rises the relative humidity close to the Peltier surface and POFBG is much higher than ambient relative humidity because higher temperature atmosphere holds more moisture. This produces a misleading temperature sensitivity.

On other hand, one can predict silica fiber grating sensor response in whole operation range since silica fiber gratings exhibit almost constant temperature/strain sensitivity. However, the different POFBG temperature/relative humidity sensitivities reported suggest that POFBG temperature/relative humidity sensitivity is not constant at different conditions. A

D. J. Webb is with Aston Institute of Photonics Technology, Aston University, Birmingham, B4 7ET, UK. (e-mail: d.j.webb@aston.ac.uk).

Copyright (c) 2018 IEEE. 
temperature sensitivity of $-55 \mathrm{pm} /{ }^{\circ} \mathrm{C}$ and a relative humidity sensitivity of $35 \mathrm{pm} / \% \mathrm{RH}$ are measured at specific environmental conditions. By knowing these values, one cannot predict the POFBG sensor response at other conditions. This makes it difficult to evaluate POFBG sensor performance and its applicability for desired applications. A general expression of POFBG temperature and relative humidity sensing response would describe POFBG performance in whole operation range and needs to be established in the first place for real application where environmental condition is always changing. However, such an investigation has not been reported.

\section{Principle Of POFBG Temperature Sensing}

The Bragg wavelength of a fiber grating depends on the effective core index, $n_{\text {eff, }}$ and the grating pitch, $\Lambda$, both of which are environment dependent in the case of a POFBG. For a POFBG the Bragg wavelength can then be expressed

$$
\lambda_{B}=2 n_{\text {eff }}(T, H) \Lambda(T, H)
$$

where $T$ is the temperature, $H$ the relative humidity. At a constant relative humidity, the Bragg wavelength change as a result of varying temperature, can be expressed as

$$
\Delta \lambda_{B}=\lambda_{0}(\alpha+\xi) \Delta T
$$

while for constant temperature, the Bragg wavelength change of a POFBG against relative humidity can be expressed as

$$
\Delta \lambda_{B}=\lambda_{0}(\eta+\beta) \Delta H
$$

where $\lambda_{0}$ is the initial Bragg wavelength, $\alpha$, the thermal expansion coefficient (TEC), $\xi$, the thermo-optic coefficient (TOC), $\eta$ the normalized refractive index change with relative humidity, and $\beta$ the swelling coefficient related to relative humidity induced volumetric change.

Silica fiber gratings exhibit constant temperature and strain sensitivity since the photo elastic properties of silica optical fibers have no significant change within the range of applied temperature and strain [10]. In the case for POFBG, as described by (2) and (3) POFBG wavelength response depends on the coefficients of $\alpha, \beta, \xi$, and $\eta$. All these four coefficients are not constant but the function of temperature and relative humidity. This can be estimated based on PMMA performance [11].

The optical and mechanical properties of PMMA have been extensively studied [12-15]. Watanabe et al [12] gave a comprehensive measurement of PMMA refractive index at different temperature and relative humidity, which shows that the PMMA refractive change with humidity and the swelling coefficient are temperature dependent. It also indicates that the TOC varies almost linearly with the temperature at different relative humidity. The TEC of PMMA also has been studied at several different conditions. Waxler et al [13] measured the thermal expansion of PMMA at dry condition. The followed studies $[14,15]$ indicated that the PMMA TEC varies linearly with relative humidity at constant temperature. All these suggest that the sensitivities of a POFBG are not constant but temperature and relative humidity dependent. Nevertheless, a systematical study of these four coefficients of PMMA over temperature and humidity change has not been reported.

\section{POFBG RESPONSES AND EXPRESSION}

The temperature and relative humidity responses of POFBGs made of different PMMA based fibers are investigated. Since the Bragg wavelength of a POFBG is a function of both temperature and relative humidity, the POFBGs were placed inside an environmental chamber (Sanyo Gallenkamp) so as to operate at the desired temperature and relative humidity. POFBG was fabricated by attaching a several centimeters length of POF that was pre-annealed in an oven at $80^{\circ} \mathrm{C}$ for 7 hours, to a single-mode silica fiber down-lead using UV curable glue. The PMMA based POF contained a 5-mm long FBG, fabricated by illuminating from above a phase mask placed on top of the POF using $325 \mathrm{~nm}$ UV light from a HeCd laser. A broadband light source with a total output optical power of 10 $\mathrm{mW}$ was used, launching light via a fiber circulator, into the POFBG under investigation. The reflection of the POF grating was monitored by using an IBSEN I-MON 400 wavelength interrogation system.

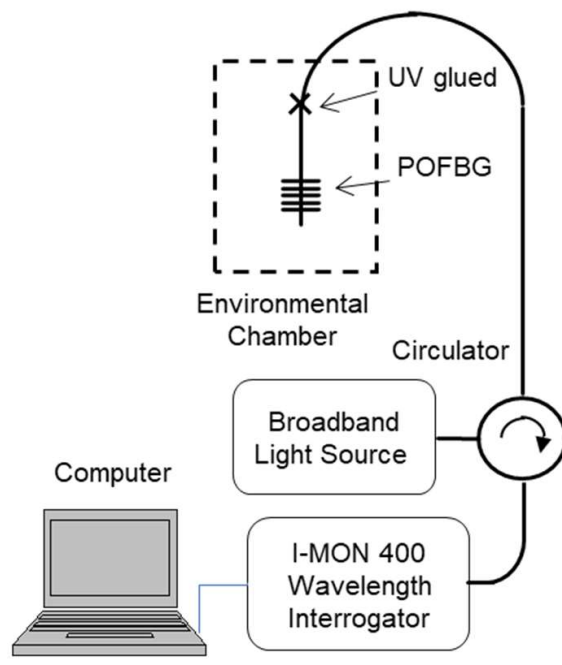

Fig. 1 Experimental arrangement for investigating POFBG responses

POFBG was placed in the chamber under varying temperature/relative humidity while relative humidity/ temperature was fixed at different levels, to investigate the temperature/relative humidity response of the POFBG sensor. The environmental chamber offers the limited range for relative humidity at the room temperature and below. Therefore, in the experiments the environmental chamber the temperature was set from $\sim 15^{\circ} \mathrm{C}$ to $40^{\circ} \mathrm{C}$ and the relative humidity was step changed from $40 \%$ to $90 \%$.

The recoded responses of a POFBG sensor are shown in Fig. 2 while the temperature of the environmental chamber was stepchanged from $14^{\circ} \mathrm{C}$ to $42^{\circ} \mathrm{C}$ at the relative humidity of $40 \%$, $55 \%, 70 \%, 85 \%$, respectively. Note that in the recoded response for $40 \% \mathrm{RH}$ the temperature started at $22^{\circ} \mathrm{C}$, due to the difficulty of environmental chamber in reaching lower temperature at such RH level. 


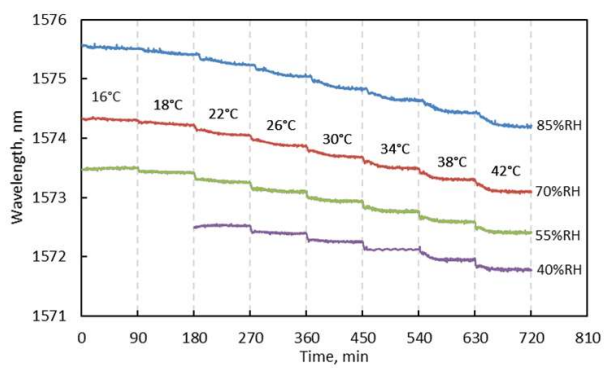

Fig. 2 Captured wavelength responses of a POFBG sensor

The POFBG wavelengths at equilibrium are plotted against the corresponding temperature and relative humidity, as shown in Fig. 3.

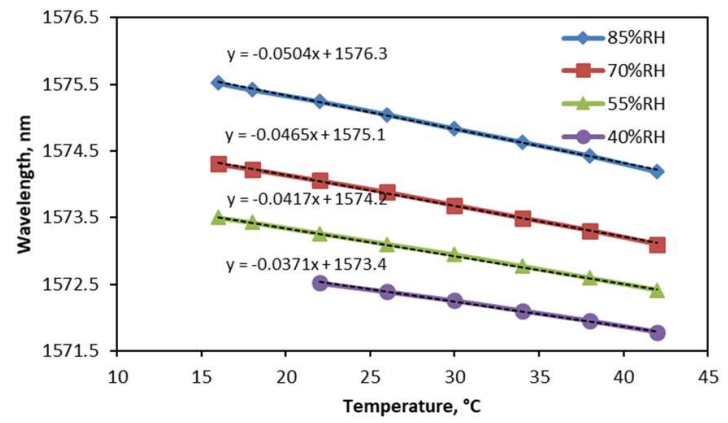

Fig. 3 POFBG wavelengths varying with temperature at different relative humidity level

It can be seen that at each relative humidity setting the POFBG temperature response shows a good linearity. The POFBG wavelength can be expressed as a linear function of temperature at each relative humidity level,

$$
\lambda_{B}=\frac{d \lambda(H)}{d T} T+\lambda_{0}(H)
$$

where $d \lambda(H) / d T$ is the temperature sensitivity at the relative humidity of $H, \lambda_{0}(H)$ the sensor wavelength at $0^{\circ} \mathrm{C}$ and the relative humidity of $H$. The equations of the trend lines in Fig. 3 have an R-squared value better than 0.998. The sensor performance approximated by the trend lines is summarized in Table I.

TABLE I

SUMMARIZED PERFORMANCE OF A POFBG SENSOR

SUMMARIZED PERFORMANCE OF A POFBG SENSOR
\begin{tabular}{|c|c|c|c|c|}
\hline$H(\% \mathrm{RH})$ & 40 & 55 & 70 & 85 \\
\hline$\frac{d \lambda}{d T}\left(\mathrm{pm} /{ }^{\circ} \mathrm{C}\right)$ & -37 & -42 & -47 & -50 \\
\hline$\lambda_{0}(\mathrm{~nm})$ & 1573.4 & 1574.3 & 1575.1 & 1576.3 \\
\hline
\end{tabular}

From Table I we can further express $d \lambda / d T$, and $\lambda_{0}$ as the function of temperature, respectively,

$$
\begin{gathered}
\frac{d \lambda}{d T}=-0.0298 H-0.0253 \\
\lambda_{0}=5.733 H+1570.75
\end{gathered}
$$

Replacing (5) into (4) we can have,

$$
\lambda_{B}=-0.0298 H T-0.0253 T+5.733 H+1571.117
$$

By now we obtain, for the first time, a general expression of the POFBG sensing response as a function of temperature and relative humidity. Differentiating (6) with respect to $H$ and $T$ leads to the POFBG relative humidity sensitivity,

$$
\frac{d \lambda_{B}}{d H}=-0.0298 T+5.733
$$

and temperature sensitivity,

$$
\frac{d \lambda_{B}}{d T}=-0.0298 H-0.0253
$$

Based on (7) and (8) one can get the POFBG relative humidity and temperature sensitivity at any given condition. For an environment of $25^{\circ} \mathrm{C}$ and $50 \% \mathrm{RH}$, the POFBG has a relative humidity sensitivity of $50 \mathrm{pm} / \% \mathrm{RH}$, and a temperature sensitivity of $-40 \mathrm{pm} /{ }^{\circ} \mathrm{C}$. These obtained figures, however, are considerably deviated from the estimated sensitivities based on bulk PMMA performance [16, 17].

In the polymer fiber drawing process, the polymer matrix undergoes a chain stretching along the fiber axis. This will orient the polymer molecules along the drawing (axial) direction. This gives rise to optical anisotropy of the fiber in the form of transverse birefringence [18] and mechanical anisotropy in the form of volume expansion [19]. The stress related anisotropy may vary with environmental conditions, leading to varying sensitivity of a POFBG sensor. Annealing POF can mitigate this effect. However, to eliminate this problem the annealing temperature needs to be raised above the PMMA transition temperature [20], which may eventually damage the fiber or the grating.

Pre-straining a POFBG can get rid of this uncertainty at the cost of reduced relative humidity sensitivity [16], while an increased sensitivity in temperature sensing can be achieved [17].

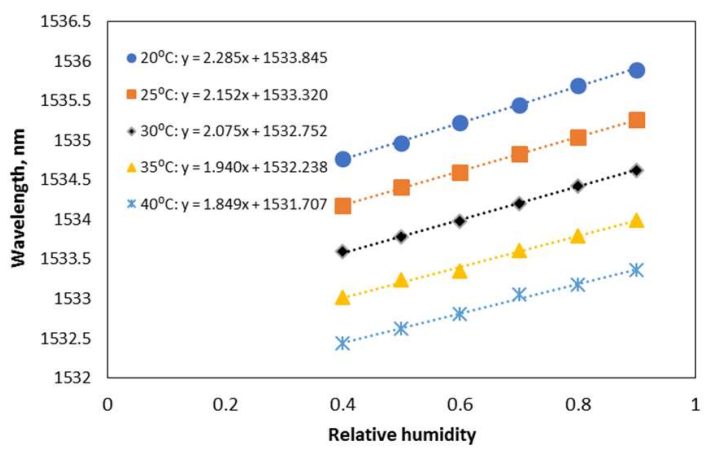

Fig. 4 The wavelengths of a pre-strain POFBG varying with relative humidity at different temperature

Fig. 4 shows the response of a pre-strained POFBG against $\mathrm{RH}$ at different temperature. Similarly, the POFBG wavelength can be expressed as a linear function of relative humidity at each temperature,

$$
\lambda_{B}=\frac{d \lambda(T)}{d T} H+\lambda_{0}(T)
$$

The response shown in Fig. 4 is summarized in Table II.

TABLE II

SUMMARIZED PERFORMANCE OF A PRE-STRAINED POFBG SENSOR

\begin{tabular}{|c|c|c|c|c|c|}
\hline$T\left({ }^{\circ} \mathrm{C}\right)$ & 20 & 25 & 30 & 35 & 40 \\
\hline$\frac{d \lambda}{d H}(\mathrm{pm} / \% \mathrm{RH})$ & 22.9 & 21.5 & 20.7 & 19.4 & 18.5 \\
\hline$\lambda_{0}(\mathrm{~nm})$ & 1533.8 & 1533.3 & 1532.8 & 1532.2 & 1531.7 \\
\hline
\end{tabular}

Then we can have,

$$
\begin{gathered}
\frac{d \lambda}{d H}=-0.0217 T+2.71 \\
\lambda_{0}=-0.1075 T+1570.75
\end{gathered}
$$


Finally, we have,

$$
\lambda_{B}=-0.0217 H T-0.1075 T+2.71 H+1536
$$

The above equation is the general expression of the POFBG response shown in Fig. 4. For an environment of $25^{\circ} \mathrm{C}$ and $50 \% \mathrm{RH}$, it indicates a relative humidity sensitivity of 21.7 $\mathrm{pm} / \% \mathrm{RH}$, and a temperature sensitivity of $-118 \mathrm{pm} /{ }^{\circ} \mathrm{C}$. These figures are very close to that estimated on PMMA performance $[16,17]$.

\section{DISCUSSION}

It needs to be pointed out that either (6) or (11) is a general expression of a POFBG response in a defined range of environmental variation. It is based on the fact that the wavelength response of a POFBG varies linearly with temperature/relative humidity at a fixed relative humidity/temperature. It has been reported that POFBG response exhibits nonlinearity [21]. This nonlinearity mainly arises from the mechanical responses of PMMA based optical fiber such as swelling and thermal expansion, which is closely related to the polymer fiber drawing process. The larger nonlinearity of response can be observed in un-annealed POFBG [22]. Although a more complicated expression could be obtained by using similar method described in Section III, this drawing process induced nonlinearity may vary with environmental condition, resulting in additional uncertainty. Pre-straining a POFBG can remove the contribution from both swelling and thermal expansion, therefore effectively overcome this nonlinearity. Furthermore, this general expression is obtained by regressing the measured sensor responses at discrete temperature/relative humidity settings. Any glitch in measured sensor responses due to different factors makes contribution to the general expression and distributes the error to the whole measurement range. This effect can only be minimized by accurately measuring and analyzing more sensor responses.

\section{CONCLUSION}

We have systematically investigated the POFBG responses over temperature and relative humidity. Temperature/relative humidity sensitivity of POFBG is found being the function of relative humidity/ temperature. We also demonstrated that a general expression of POFBG wavelength response against temperature and relative humidity can be obtained. This will help to predict sensor performance and define the operation range for real applications.

\section{REFERENCES}

[1] D. J. Webb, and K. Kalli, "Polymer Fiber Bragg Gratings" in Fiber Bragg Grating Sensors, A. Cusano, A. Cutolo and J. Albert ed., Bentham Science Publishers Ltd, (2011)

[2] C. Zhang, W. Zhang, D. J. Webb, G.-D. Peng, "Optical fiber temperature and humidity sensor," Electron. Lett., vol. 6, no. 9, pp. 643-644, 2010

[3] N. G. Harbach, "Fiber Bragg gratings in polymer optical fibers," Ph.D. dissertation, Inst. OPT Lab, EPFL, Lausanne, Switzerland 2008.
[4] W. Zhang, D. J. Webb and G.-D. Peng, "Polymer optical fiber Bragg grating acting as an intrinsic biochemical concentration sensor," Opt. Lett. vol. 37, no. 8, pp. 1370-1372, 2012.

[5] C. Zhang, X. Chen, D. J. Webb, and G.-D. Peng, "Water detection in jet fuel using a polymer optical fibre Bragg grating," Proc. SPIE, vol. 7503, p. 750380 , Oct. 2009

[6] W. Zhang, D. J. Webb, "PMMA Based Optical Fiber Bragg Grating for Measuring Moisture in Transformer Oil," IEEE Photon. Tech. Lett., vol. 28, no. 21, pp.2427-2430, 2016.

[7] H. B. Liu, H. Y. Liu, G. D. Peng, and P. L. Chu, "Strain and temperature sensor using a combination of polymer and silica fibre bragg gratings," Opt. Commun. vol. 219, pp. 139-142, 2003.

[8] G. N. Harbach, Hans G. Limberger, and René P. Salathé, "Influence of Humidity and Temperature on Polymer Optical Fiber Bragg Gratings," Proc. Bragg Gratings, Photosensitivity, and Poling in Glass Waveguides, BTuB2, 2010.

[9] H. Y. Liu, G. D. Peng, and P. L. Chu, "Thermal tuning of polymer optical fiber bragg gratings," IEEE Photon. Technol. Lett. vol. 13, no. 8, pp. 824$826,2001$.

[10] A. Barlow, D. Payne, "The stress-optic effect in optical fibers," IEEE J Quantum Electron., vol. 19, no. 5, pp. 834-839, 1983.

[11] W. Zhang, D. J. Webb, and G. D. Peng, "Investigation into time response of polymer fiber Bragg grating based humidity sensors," J. Lightw. Technol., vol. 30, no. 8, pp. 1090-1096, Apr. 15, 2012.

[12] T. Watanabe, N. Ooba, Y. Hida, and M. Hikita, "Influence of humidity on refractive index of polymers for optical waveguide and its temperature dependence," Appl. Phys. Lett., vol. 72, no. 13, p. 1533, 1998.

[13] Roy M. Waxler, Deane Horowitz, and Albert Feldman, "Optical and physical parameters of Plexiglas 55 and Lexan", Appl. Opt., vol. 18, no. 1, pp. 101-104, 1979.

[14] O. V. Startsev, V. P. Rudnev, "Reversible moisture effects in the climatic ageing of organic glass," Polym. Degrad. Stab. 39 373-379, 1993.

[15] W. D. Drotning, E. P. Roth, "Effects of moisture on the thermal expansion of poly(methyl methacrylate)," J. Mater. Sci. vol. 24, no. 9, pp. 3137$3140,1989$.

[16] W. Zhang and D. J. Webb, "Humidity responsivity of poly(methyl methacrylate)-based optical fiber Bragg grating sensors," Opt. Lett., vol. 39, no. 10, pp. 3026-3029, 2014.

[17] W. Zhang, D. J. Webb and G. -D. Peng, "Enhancing the sensitivity of poly(methyl methacrylate) based optical fiber Bragg grating temperature sensors," Opt. Lett. vol. 40, no. 17, pp. 4046-4049, 2015.

[18] P. Ji, A. D. Q. Li and G. D. Peng, "Transverse birefringence in polymer optical fibre introduced in drawing process," Proc. of SPIE, Vol. 5212, 108-116, (2003)

[19] L. -H. Wang, C. L. Choy, and R. S. Porter, "Thermal Expansion of Oriented Poly(methy1Methacrylate)," J. Polym. Sci., vol. 21, no. 4, pp. $657-665,1983$

[20] C. Jiang, M. G. Kuzyk, J. Ding, W. E. Johns, and D. J. Welker, "Fabrication and Mechanical Behavior of Dye-Doped Polymer Optical Fiber," J. Apl. Phys. vol. 92, no. 1, pp. 4-12, 2002.

[21] W. Zhang. D. J. Webb, G.-D. Peng, "Nonlinear response in polymer optical fibre Bragg grating based sensors," Proc. of SPIE, Vol. 8421, $84214 \mathrm{~N}-4,2012$

[22] W. Zhang. D. J. Webb, "Factors influencing temperature sensitivity of PMMA based optical fiber Bragg gratings," Proc. SPIE, vol. 9128, pp. 91280M-1-91280M-7, May 2014 
2018-12-25

General expression of poly(methyl

methacrylate) optical fiber Bragg grating sensing response

\section{Zhang, Wei}

IEEE

W. Zhang and D. J. Webb. General expression of poly(methyl methacrylate) optical fiber Bragg grating sensing response. IEEE Photonics Technology Letters, Volume 31, Issue 3, February 2019, 234-237

https://doi.org/10.1109/LPT.2018.2889758

Downloaded from Cranfield Library Services E-Repository 